\title{
Justicia de Paz en el Perú: Una instancia de resolución de conflictos que garantiza el acceso a la justicia
}

\author{
Fernando Valverde Caman \\ Caman4@hotmail.com \\ https://orcid.org/0000-0001-8550-8556 \\ Académico de la Universidad Peruana de Ciencias Aplicadas
}

\section{RESUMEN}

La presente investigación analiza la labor fundamental que realizan los jueces de paz en el Perú, ubicada en el primer nivel de la administración de justicia. Ellos son conocidos como los "jueces conciliadores" y están legitimados por las comunidades al ser elegidos entre sus miembros para que resuelvan los conflictos sociales de menor trascendencia jurídica y social, impartiendo justicia desde conflictos familiares, propiedad, tierras, faltas menores, entre otros. Su reconocimiento y garantía que tienen los pobladores en este tipo de justicia es por la accesibilidad y la rapidez en la solución obtenida del conflicto y la restauración de la armonía social. El estudio permitió conocer las funciones que realizan los jueces de paz, los criterios, valores, usos, costumbres y su leal saber y entender al momento de resolver los conflictos para el mantenimiento de la paz social.

Palabras clave: Jueces de paz, conciliación, resolución de conflictos sociales, acceso a la justicia, costumbres, paz social. 


\title{
Justice of Peace in Peru: An instance of conflict resolution that guarantees access to justice.
}

\begin{abstract}
This research analyzes the fundamental work performed by justices of the peace in Peru, located at the first level of the administration of justice. They are known as the "conciliatory judges" and are legitimized by the communities when they are elected from among their members to resolve social conflicts of less legal and social significance, dispensing justice from family conflicts, property, land, minor offenses, among others. Its recognition and guarantee that the inhabitants have in this type of justice is due to the accessibility and speed in the solution obtained from the conflict and the restoration of social harmony. The study allowed us to know the functions performed by justices of the peace, the criteria, values, customs, and their loyal knowledge and understanding when resolving conflicts for the maintenance of social peace.
\end{abstract}

Keywords: Justices of the peace, conciliation, resolution of social conflicts, access to justice, customs, social peace.

Artículo recibido: 19 enero 2020 Aceptado para publicación: 22 febrero Correspondencia: caman4@ hotmail.com Conflictos de Interés: Ninguna que declarar 


\section{INTRODUCCIÓN}

La presente investigación estudia la institución de la Justicia de Paz en el Perú, entendida ésta como el primer nivel en la que se imparte justicia dentro del Poder Judicial. Ellos son conocidos como "jueces conciliadores" y están legitimados por las comunidades, al ser elegidos por elección popular entre sus miembros para que resuelvan los conflictos sociales básicos en nombre del Estado. Con relación a la función que cumplen los jueces de paz, Ticona señala "Los jueces de paz, ante todo, cumplen una función conciliadora, pero cuando no es posible conciliar están facultados para expedir sentencias. Pero tales sentencias se pronuncian según el leal saber y entender del juez de paz, preservando siempre los valores que la Constitución Política consagra y respetando la cultura y las costumbres de su comunidad". (Ticona, 2015, p. 7) De ahí la importancia también de estudiar los conflictos más frecuentes y la percepción que tiene los usuarios de los jueces en esta instancia judicial.

\section{REALIDAD SOCIO JURIDICA DE LA JUSTICIA DE PAZ}

La Justicia de Paz es reconocida como una instancia no formal de resolución de conflictos en el ámbito local y vecinal, basada en la conciliación y la equidad, que respeta las características de pluriculturalidad de nuestro país y contribuye a lograr que la justicia no sea un factor de exclusión y a superar las barreras geográficas, económicas, lingüísticas y culturales que la afectan. De ahí que la justicia de paz, conocida como justicia no letrada o justicia conciliadora, constituye uno de los principales mecanismos a través de los cuales los ciudadanos canalizan sus conflictos individuales tanto en el ámbito urbano como en el rural ${ }^{2}$.

Su marco jurídico está contenido en el artículo $152^{\circ}$ de la Constitución Política del Estado, los artículos $61^{\circ}$ y siguientes de la Ley Orgánica del Poder Judicial; el Reglamento de Jueces de Paz de 1854 (derogada) oficialmente el 3 de enero del 212 entrando en vigor la Ley de justicia de Paz (Ley $29824)^{3}$.

\footnotetext{
${ }^{1}$ Programa de las Naciones Unidas para el Desarrollo (PNUD). (2015). Fortaleciendo la Justicia de Paz en el Perú. Recuperado de: https://www.pe.undp.org/content/peru/es/home/library/democratic governance/fortaleciendo-lajusticia-de-paz-en-el-peru.html [Consulta: 4 de febrero 2020].
}

${ }^{2}$ Informe PNUD. Fortaleciendo la justicia de paz en el Perú. (2015). "En el Perú, la justicia de paz, justicia consuetudinaria y pluricultural, es reconocida constitucionalmente como un fuero que sirve de primer escalón en la Administración de Justicia. Por ello, cumple un rol fundamental en la consolidación del Estado democrático de Derecho al brindar a las personas que viven en zonas rurales y urbano-marginales la posibilidad de acceder a mecanismos que respetan su idiosincrasia, y permite a la institucionalidad del Poder Judicial la posibilidad de ampliar y reforzar su presencia en el territorio nacional".

${ }^{3}$ Tras más de siglo y medio de vigencia, el 3 de enero de 2012 se derogó oficialmente el Reglamento de Jueces de Paz de 1854, y en su reemplazo se promulgó la vigente Ley de Justicia de Paz (Ley 29824). 
Además la justicia de paz es desempeñada por personas que no necesariamente son abogados o "entendidos en leyes", sino por campesinos, pequeños comerciantes, profesores, jubilados, entre otros, que están facultados por ley para resolver determinados conflictos familiares, patrimoniales y comunitarios que se puede observar que estos conflictos son menores, pero que para la comunidad son fundamentales para la convivencia y para mantener la paz social con justicia en muchas comunidades rurales o urbano marginales.

A nivel nacional existe en el Perú 5,930 Jueces de Paz, la mayoría ubicados en las zonas rurales, pero también ejercen justicia en las principales ciudades. La ley les reconoce competencia para casos de deudas y conflictos patrimoniales hasta 3,200 soles. De igual forma conoce de faltas personales, violencia familiar y algunos trámites notariales. En todos los asuntos que conozcan de una controversia están autorizados por la Constitución y la Ley Orgánica del Poder Judicial para resolver de acuerdo con sus propios criterios. Costumbres y valores de justicia, pero siempre respetando y garantizando los derechos humanos de los justiciables.

Actualmente, en muchos lugares del Perú, la mayoría de los conflictos son atendidos por los Jueces de Paz. Para los sectores más pobres de la población, los vecinos entienden que son las únicas autoridades judiciales a los que pueden acudir y los que tienen mayor legitimidad social. Debe tenerse presente que, los nuevos cambios poblacionales, como la migración y la urbanización y otros fenómenos sociales como la expansión del sistema educativo han generado una marcada diferenciación entre los actuales Jueces de Paz en el Perú y de los que venían desempeñando y que desarrollaremos más adelante.

Tal como referíamos los datos actualizados recogidos al 2019 y actualizados señalan una cifra a nivel nacional de 5,930 ${ }^{4}$ juzgados de paz, de acuerdo con información brindada por las Cortes Superiores a la Gerencia General del Poder Judicial. Sin embargo, esta cantidad, como otras, presenta que los juzgados formalmente establecidos son registrados por dichas cortes, pero que constantemente varían por el ejercicio en funciones, de renuncia o traslado de los jueces a otras zonas.

Si analizamos las zonas donde se habría dado aumento podemos apreciar que ellas fueron espacios de fuerte violencia política (como Ayacucho, Cuzco, San. Martín y Huancavelica), lo que podría llevar a suponer que la mayor parte de esta cifra son juzgados que han retomado sus funciones luego del proceso de violencia política.

\footnotetext{
${ }^{4}$ Informe PNUD. Fortaleciendo la justicia de paz en el Perú (2015)
} 
En la actualidad podemos destacar el siguiente mapa judicial de los juzgados de paz a nivel nacional conforme al cuadro elaborado por la Gerencia de Planificación del Poder Judicial.

Cuadro No 1: Juzgados de Paz por Distrito Judicial año 2019

\begin{tabular}{|l|l|l|}
\hline $\begin{array}{l}\text { Distrito Judicial } \\
\mathbf{N}^{\circ} \% \text { Distrito Judicial }\end{array}$ & $\mathbf{N}^{\circ}$ & $\mathbf{\%}$ \\
\hline Cajamarca & 497 & $8.38 \%$ \\
\hline Ancash & 460 & $7.76 \%$ \\
\hline Puno & 437 & $7.37 \%$ \\
\hline Huánuco & 420 & $7.08 \%$ \\
\hline Junín & 419 & $7.07 \%$ \\
\hline Ayacucho & 399 & $6.73 \%$ \\
\hline Cusco & 352 & $5.94 \%$ \\
\hline Lambayeque & 337 & $5.68 \%$ \\
\hline Arequipa & 235 & $3.96 \%$ \\
\hline La Libertad & 228 & $3.84 \%$ \\
\hline Huancavelica & 225 & $3.79 \%$ \\
\hline Apurímac & 222 & $3.74 \%$ \\
\hline San Martín & 187 & $3.15 \%$ \\
\hline Amazonas & 183 & $3.09 \%$ \\
\hline Piura & 172 & $2.90 \%$ \\
\hline Loreto & 134 & $2.26 \%$ \\
\hline Huaura & 114 & $1.92 \%$ \\
\hline Cañete & 102 & $1.72 \%$ \\
\hline Sullana & 92 & $1.55 \%$ \\
\hline Santa & 90 & $1.52 \%$ \\
\hline Selva Central & 89 & $1.50 \%$ \\
\hline Moquegua & 83 & $1.40 \%$ \\
\hline Ica & 71 & $1.20 \%$ \\
\hline Lima Este & 71 & $1.20 \%$ \\
\hline Pasco & 58 & $0.98 \%$ \\
\hline Tacna & 58 & $0.98 \%$ \\
\hline Ucayali & 57 & $0.96 \%$ \\
\hline Madre de Dios & 45 & $0.76 \%$ \\
\hline Tumbes & 30 & $0.51 \%$ \\
\hline Lima Norte & 28 & $0.47 \%$ \\
\hline Ventanilla & 26 & $0.44 \%$ \\
\hline Callao & 5 & $0.08 \%$ \\
\hline Lima Sur & $\mathbf{5 9 3 0}$ & $0.07 \%$ \\
\hline Lima & $\mathbf{1 0 0 . 0 0 \%}$ \\
\hline TOTAL & (2) \\
\hline
\end{tabular}

Fuente: Resoluciones Administrativas del Consejo Ejecutivo del Poder Judicial 2001-2019, publicadas en el diario El Peruano. Elaboración: Gerencia de Planificación / Sub Gerencia de Estadística 


\section{TENDENCIAS DE LA JUSTICIA DE PAZ EN EL PERÚ}

Tal como lo venimos señalando en el Perú, la mayor parte de conflictos jurídicos que llegan ante el Poder Judicial no son resueltos por magistrados formados en el campo del derecho, sino por vecinos elegidos en sus comunidades al que pertenecen tomando en cuenta sus cualidades personales y el liderazgo que tienen en la población. De acuerdo con el último mapa judicial señalado líneas arriba en nuestro país, la Justicia de Paz sigue teniendo una presencia en las zonas rurales, con una significativa presencia en la región andina. El resto de los jueces se divide, entre la costa y la región amazónica. Estas diferencias son muy importantes, por cuanto la labor de la Justicia Paz se caracteriza por la heterogeneidad y las diferencias culturales por regiones en donde están presentes impartiendo justicia.

A pesar de su importancia a escala nacional, la Justicia de Paz no cuenta con un apoyo optimo en la labor que ejercer como parte del Estado y del propio Poder Judicial. Los jueces de paz, en su mayoría, no reciben ningún apoyo logístico ni en infraestructura. Tampoco han recibido capacitación relacionada con el rol que desempeñan, lo que determina que, en esta tarea, debe seguir fortaleciéndose para optimizar mejor el desempeño de la justicia. La Justicia de Paz, conocida por mucho tiempo como Justicia de Paz No Letrada, es una institución que, nació con la República. Y ella ha estado presente desde la Constitución de 1823, y en las subsiguientes constituciones que nos han regido en la república, hasta la vigente de 1993. Desde entonces, ha evolucionado y cambiado sobre todo en las fórmulas para designar a quienes ejercen tales cargos, pero en esencia mantiene su concepción de ejercer una justicia vecinal, popular y rápida en la solución de conflictos, y que busca recuperar o mantener la armonía en base a la conciliación. Es por ello que el Juez de paz no es necesariamente un abogado, o profesional por lo que es un vecino, respetado y con influencia moral y de liderazgo en su comunidad para que pueda cumplir un papel de conciliador de las diferencias o pleitos vecinales que se presenten. La Ley Orgánica del Poder Judicial señala como únicos requisitos para ocupar estos cargos, ser peruano de nacimiento, tener una conducta intachable, ser ciudadano en ejercicio, ser vecino del lugar, tener profesión u oficio conocido, haber terminado por lo menos la educación primaria, y hablar el idioma predominante en el lugar (quechua, aymara, ashaninka, etc.).

Los jueces de paz no están obligados a fundamentar jurídicamente sus resoluciones, por la que pronuncie se dará "según su leal saber y entender", debidamente motivadas, según el texto de la LOPJ. Impartir justicia significa que los Jueces de Paz deberá resolver los conflictos que se presentan en las comunidades del cual son conocedores de su propia realidad, tomando en cuenta 
los valores, las costumbres del lugar, prevaleciendo el derecho consuetudinario y utilizando su propio criterio. La aplicación de su propio razonamiento debe tener como límite el respeto a los derechos humanos fundamentales conforme lo señala la Constitución. Por estas razones, el Poder Judicial capacita permanentemente, con el apoyo de otras instituciones, a los Jueces de Paz en el país, en temas de conciliación, violencia familiar, derecho de personas, derecho consuetudinario y criterios de equidad.

El Perú es un país fragmentado y heterogéneo geográfica y culturalmente, por lo que los Jueces de Paz constituyen el primer contacto de la población con la justicia, y es además la justicia más cercana que conocen, al funcionar la institución en comunidades pequeñas donde el juez es una persona que vive en el mismo lugar. Es por lo que una de sus características principales es la rapidez en el acceso a la justicia con que se resuelven los conflictos, recurriendo al uso de la conciliación para preservar la paz social. Cabe señalar que la Justicia de Paz goza de prestigio entre la población y tiene importantes niveles de confiabilidad entre los pobladores. Los usuarios no tienen que caminar grandes distancias para encontrar a la autoridad judicial pues generalmente llegan caminando hasta la sede del Juzgado de Paz, que en un gran porcentaje es la vivienda misma del Juez o en otros pocos casos un local cedido por la municipalidad del lugar. La Justicia de Paz integra el Poder Judicial y ocupa casi el 73\% del número total de jueces (los magistrados profesionales sólo ocupan el $27 \%$ de este universo). Eso nos puede dar una dimensión de lo que hablamos cuando nos referimos a la justicia de paz. ${ }^{5}$

Otro aspecto importante a resaltar es la mayor aceptación de la justicia de paz entre los miembros de la comunidad. Hans-Jurgen Brandt señalaba en 1990 que el índice de aprobación de la justicia de paz era de $63.2 \%{ }^{6}$. Más recientemente en el citado estudio de IDL, se ratifica esta percepción mayoritariamente favorable, pues una encuesta aplicada revela que el 64.66\% de la población está satisfecha con el juez de paz. Ello contrasta con la opinión crítica y deficitaria que tiene la ciudadanía respecto de la justicia profesional. Por lo expuesto podemos afirmar que los jueces de paz son esencialmente conciliadores y se reconocen como facilitadores para la resolución de conflictos y se reafirma la garantía del acceso a la justicia en la solución rápida. Esta actitud es

5 Fuente: Cortes Superiores de Justicia. Sub Gerencia de Estadística. Lima, octubre del 2002. La metodología usada para esta estadística refiere que la información fue proporcionada por cada Presidente de Corte y cotejada por la Sub Gerencia en forma telefónica, para confirmar los datos. De ese total, el documento informa que 223 jueces de paz no han sido designados o han renunciado. Los distritos judiciales que presentan mayor incidencia de vacíos en las plazas son de Ayacucho (17\%), Cajamarca (12\%) y Apurímac (11\%).

6 Brandt, Hans-Jurge. "En nombre de la Paz Comunal. Un análisis de la Justicia de Paz en el Perú. Fundación Friedrich Naumann. 1990. p. 385. 
cualitativamente distinta de la que normalmente adoptan los jueces profesionales, quienes recurren mediante la "aplicación de leyes". Esta facultad conciliadora que tienen los jueces de paz es reconocida también por nuestra legislación y es, precisamente, por esta vía que los jueces pueden resolver muchas materias que la legislación les limita. Por ello, pueden resolver casos de violencia familiar, alimentos, tenencias de menores, pago de soles, entre otras materias. Esta práctica también puede explicar la rapidez con lo que se resuelven los asuntos que las partes, ponen a su consideración.

\section{LA JUSTICIA DE PAZ Y SU LABOR CONCILIADORA}

Conforme al artículo $64^{\circ}$ del Decreto Supremo $N^{\circ}$ 017-93-JUS, Texto Único Ordenado de la Ley Orgánica del Poder Judicial, el juez de paz es esencialmente un juez de conciliación. Se encuentra facultado para acercar a las partes alternativas de solución sin imponer un acuerdo. De no lograrse la conciliación, el juez de paz tiene la potestad de conocer solamente cierto tipo de causas, como son los procesos en materia de alimentos, de pago de dinero y otros conforme a ley, dentro de determinadas cuantías. De este modo, formalmente la cobertura que brinda la justicia de paz es restringida. Sin embargo, todos los estudios realizados señalan que, en los hechos, las causas que son de conocimiento de los jueces de paz suelen ser más amplio que las establecidas por ley, sea por decisión propia del juez o por presión social.

Esta situación ha llevado a un dilema que aún no es resuelto debidamente: ¿deben ampliarse las materias a ser vistas por el juez de paz, a fin de adecuar la norma a la realidad, o deben controlarse mejor las causas que ingresan a esta instancia, adaptando así la realidad a la norma? A nuestro entender, una respuesta adecuada pasa por una solución intermedia, que reconociendo mayores facultades a la justicia de paz no implique el desconocimiento de los derechos ciudadanos, tema que debe merecer un debate apropiado.

Los tipos de conciliación en la justicia de paz consisten en: a) La transacción, entendida como la conciliación en la que el juez sólo es un fedatario de un acuerdo al que las partes han arribado previamente y recurren al juez de paz para que éste simplemente dé fe de los acuerdos tomados. Esto se asemeja mucho a la estructura de lo que denominamos una transacción, es decir, acuerdos o compromisos que las partes adoptan por propia iniciativa y voluntariamente. b) La conciliación estándar, es aquella en la que efectivamente el juez de paz cumple un rol mediador, sea acercando a las partes, sea formulando propuestas de alternativas de solución y c) La conciliación impuesta, se da cuando el juez de paz "impone" una solución del conflicto a las partes, aceptando y firmando el 
acta definitiva. Este tipo de actas se utiliza esencialmente en los casos de faltas, y tienen la finalidad de revestir de cierta forma consensual a las penas que ellos imponen.

Valorando las implicancias de la resolución de conflictos en la Justicia de Paz ésta tiene otras finalidades y valores que la Justicia Profesional. Mientras en la justicia ordinaria la finalidad en los juicios penales radica en la sanción, la corrección, la prevención y la resocialización. La finalidad de los juicios civiles en la Justicia de Paz es la búsqueda del restablecimiento de las relaciones interpersonales, la resolución privada de los conflictos menos graves. En la Justicia de Paz se busca la reconciliación de las partes en conflicto y restauración de la armonía social en la comunidad. Existe consenso entre los investigadores respecto a la rapidez de la justicia en esta instancia para solucionar los problemas y la conciliación constituye un mecanismo eficaz. Al parecer, ella es producto de la alta informalización de la justicia de paz y del propio mecanismo de conciliación utilizado. Esto implica la inexistencia de formalidades jurídicas que limiten la actuación de los jueces de paz.

\section{FUNCIÓN JURISDICIONAL DE LOS JUECES DE PAZ}

Se han venido desarrollando diversos estudios en torno a la función jurisdiccional con la finalidad de establecer la carga procesal generada por la justicia de paz y del cual no existe datos reales confiables. Sin embargo en un estudio realizado por la Comisión Especial para la Reforma de la Justicia se destacó lo siguiente: “Al igual que en otros aspectos, no existen datos firmes y confiables acerca de la carga procesal que manejan los jueces de paz. Los datos existentes apuntan, sin embargo, a afirmar que esta carga no suele ser muy alta, lo que se ve fortalecida además con la flexibilidad y rapidez de los procesos que se producen en esta instancia de justicia. En uno de los escasos estudios sobre este tópico, Brandt estableció un promedio de 68 causas al año por juzgado de paz en la sierra y de 139.9 causas anuales en la costa, si bien el mismo investigador reconoció que ésta era una cifra muy baja"7, Sobre la base de estas cifras, Brandt dedujo que la justicia de paz tramitaba alrededor del $47 \%$ de todas las causas ingresadas en los juzgados de primera instancia en el ámbito nacional, con excepción de Lima, en tanto es este distrito judicial existen contados juzgados de este tipo. Otros estudios más focalizados, como el realizado por Luis Puentes en los juzgados de la costa norte de Piura, muestran un volumen promedio de 20 causas al mes, lo que hace un total de 240 causas al año ${ }^{8}$.

Pese a la información señalada, no conlleva a fijar un estimado claro de la carga procesal a nivel de los juzgados de paz, por la forma de funcionamiento que realizan, caracterizado por la diversidad de conflictos que conocen a nivel de esta instancia, incluso de carácter extralegal, sino

\footnotetext{
${ }^{7}$ Brandt, Hans.Jurgen. En nombre de la paz comunal. Un análisis de la justicia de paz en el Perú. Lima Fundación Friedrich Nauman, diciembre de 1990, p.29

${ }^{8}$ Puentes, Luis. Los juzgados de paz en la Costa Norte. Lima. Centro de Estudios y Publicaciones, octubre 1997 , p 369.
} 
además porque impide ver un conjunto de factores, de accesibilidad y cultural del juzgado, los costos de los procedimientos, etc. Por ello, urge definir una política judicial clara vinculada a la justicia de paz con más información de la carga procesal y el tipo de conflictos procesados en esta instancia primaria.

\section{ELECCIÓN DE JUECES DE PAZ}

Una de las novedades de la Constitución de 1993, es la norma que establece la obligatoriedad de la elección de jueces de paz. Esta cláusula constitucional ha sido desarrollada por la Ley $\mathrm{N}^{\circ} 26859$ Ley Orgánica de Elecciones- y la ley N²9824 -Ley de Justicia de Paz y el Reglamento de Elección popular de los jueces de paz - Resolución administrativa- Nº98-2012-CE-PJ.

El Reglamento destaca dentro de sus objetivos que el proceso de elección popular del juez de paz garantice la participación mayoritaria, directa y democrática de los pobladores que radican en un área geográfica en la que el juzgado de paz ejerce jurisdicción. Así mismo contempla que debe garantizar que el juez de paz sea idóneo para el cargo y el goce del reconocimiento y respeto de la población. Y tercero que busque asegurar la adecuada y correcta coordinación entre el Poder Judicial, las autoridades electorales y las autoridades locales y comunales que intervienen en el proceso de elección popular del juez de paz.

Es importante tener en cuenta que las normas de elecciones señalan que, en los casos de las comunidades campesinas y nativas, la elección de jueces de paz se realizará respetando los usos y costumbres, y la ONPE debe solamente super vigilar que las asambleas u otros métodos de elección directa expresen la real voluntad de los ciudadanos electores. Los candidatos a jueces de paz están prohibidos de postular a dos o más judicaturas simultáneamente y a realizar campaña política. Ningún partido político, agrupación independiente, grupo vecinal o cualquier otra organización formal o informal, podrá auspiciar o patrocinar a los candidatos.

En este tema, la Ley de Elecciones de Jueces de Paz, así como la Ley N²9824-Ley de Justicia de $\operatorname{Paz}^{9}$ señala entre los requisitos para ser juez de paz ser mayor de 30 años. Un requisito importante es

\footnotetext{
${ }^{9}$ Ley de Justicia de Paz: Artículo 1. Requisitos para ser juez de paz Los requisitos para ser juez de paz son los siguientes: 1. Ser peruano de nacimiento y mayor de treinta (30) años. 2. Tener conducta intachable y reconocimiento en su localidad. 3. Ser residente por más de tres (3) años continuos en la circunscripción territorial del juzgado de paz al que postula. La residencia estacional no acredita el cumplimiento del presente requisito, aunque supere los tres (3) años. 4. Tener tiempo disponible para atender el despacho y satisfacer la demanda del servicio de la población. 5. Tener ocupación conocida. 6. Conocer el idioma castellano, así como la lengua y/o los dialectos predominantes en la localidad.7. No haber sido condenado por la comisión de delito doloso.8. No haber sido destituido de la función pública. 9. No haber sido objeto de revocatoria en cargo similar. 10. No ser deudor alimentario moroso. 11. No estar incurso en ninguna incompatibilidad establecida por ley.
} 
el conocimiento del idioma local. Otro requisito de la Ley señala que no haya sido destituido de la función pública y no haya sido objeto de revocatoria en el cargo similar, así como no ser deudor alimentario moroso. En caso de que un Juez de Paz renuncie, éste enfermo, haya pedido licencia o haya fallecido, el cargo es asumido por el Juez de Paz accesitario. También puede asumir un caso específico en el que el Juez de Paz titular deba inhibirse por razones familiares o personales.

El requisito de tener conducta intachable y reconocimiento en su localidad (art. 1, inciso 2), depende de las normas y valores locales de donde se encuentra el juzgado. Por ejemplo, el adulterio podría en determinadas localidades ser motivo para no cumplir con el perfil para ser juez de paz. Es necesario tener residencia permanente, no estacional; es decir, no interrumpida, durante los tres años que establece la ley (art. 1, inciso 2). No se debe haber sido condenado por comisión de delito doloso. Esto significa que, si alguien fue condenado por haber cometido delito doloso, no puede ser juez de paz, aunque haya cumplido la condena. Es decir, el cumplimiento de condena, la rehabilitación en términos legales no habilita el acceso al cargo de juez de paz.

Dentro de los mecanismos para acceder al cargo de juez de paz la ley señal que ésta puede ser por elección popular, es decir, por voto directo de la mayoría de la población del lugar donde ejerce jurisdicción el juez de paz y por selección, es decir, que el propio Poder Judicial escoge al juez de paz a partir de las propuestas que presenta la población organizada, mediante el procedimiento dispuesto por el reglamento respectivo.

\section{EL NUEVO PERFIL DE LOS JUECES DE PAZ}

Ya desde 1999, la Comisión Ejecutiva del Poder Judicial dispuso en dos resoluciones sucesivas la realización de procesos de elección de Jueces de Paz. Esta norma no se ajustaba a lo dispuesto por la Constitución Política de 1993, pero tuvo como efecto que las Cortes Superiores promovieran los procesos de elección de jueces de paz en todo el Perú. El resultado fue que se manifestaron una serie de cambios culturales que se daban en la población y que fueron alterando el perfil de los Jueces de Paz.

Los datos demuestran que especialmente en la Costa, existe la tendencia a pasar del Juez de Paz tradicional, una persona mayor que decide los casos de acuerdo con su experiencia, a elegir Jueces de Paz más jóvenes, pero que pueden comprender mejor nuevas situaciones o dar un enfoque diferente a antiguos problemas. La edad promedio de los Jueces de Paz ha disminuido, en 1998 un tercio de los Jueces de Paz tenía más de 50 años. Actualmente, solo se puede encontrar un porcentaje similar en la Región Andina. 
De esta manera tenemos que, precisamente en las zonas de la costa, donde la esperanza de vida es más alta, la población prefiere elegir Jueces de Paz más jóvenes. Los Jueces de Paz mayores suelen tener menos instrucción especialmente en las zonas andinas, donde la mayoría sólo ha estudiado primaria. Por lo tanto, eligiendo a una persona más joven se puede garantizar que tenga mayor nivel educativo, siendo, como veremos a continuación, otro criterio importante la formación profesional. Las Resoluciones Administrativas del año 1999 establecieron una serie de normas para fomentar la presencia de mujeres en el cargo de Juez de Paz, que no tenían carácter determinante, pero que podían fomentar esta tendencia. La norma tuvo como resultado que muchas mujeres se incorporaran al cargo, especialmente en aquellos lugares más cercano a las zonas rurales. En la zona Andina el porcentaje llega al 11.6\% mientras en la Amazonía el 13\%. Inclusive en los distritos judiciales más tradicionales, donde toda la población rural es de origen indígena, como Ayacucho o Huancavelica, donde en 1998 la totalidad de Jueces eran varones, ahora tenemos un porcentaje del 10\% de mujeres. Tomando en cuenta la proporción de Jueces de Paz existente en cada región, podemos decir que en la actualidad el $14 \%$ de los jueces de paz son mujeres.

Paulatinamente la población está aceptando una autoridad femenina, especialmente los jóvenes y las mismas mujeres que podrían mantener prejuicios al respecto. Personas mayores en la zona andina se han mostrado escépticos sobre el desempeño de una mujer como Juez de Paz.

En muchos lugares, un factor clave para la elección de una mujer es el mayor nivel educativo, las mujeres tienen educación superior completa frente a los varones. La educación se convierte en un canal muy importante para adquirir ascendencia dentro de la Comunidad. En general, una vez que la mujer asume el cargo, suelen ser consideradas personas muy honestas, se indica también que son más sensibles frente a la violencia familiar y los casos de alimentos.

Se aprecia que la población tiende a elegir Juez de Paz a personas que han estudiado y tienen una mayor formación desde el punto de vista occidental. El Juez de paz tradicional, es decir, la persona cuya sabiduría deriva de su edad, está dejando pasar a un juez de paz moderno, más joven e instruido. Estos cambios en los Jueces de Paz revelan también cambios en la comunidad. Muchas veces, los cambios son más visibles en las mujeres: ellas tienen un nivel educativo muy superior al promedio de los Jueces de Paz varones. Esto revela que cuando una persona tiene una educación superior, la población está dispuesta en confiar en ella. De igual forma, cuando se elige a una persona que lleva poco tiempo en la comunidad, éste generalmente tiene instrucción superior y ha residido mucho tiempo en una ciudad grande. 


\section{CONSIDERACIONES FINALES}

La experiencia del modelo integrador adoptado por la Justicia de Paz en el Perú ha venido siendo reconocido como una instancia fundamental de administración de justicia del primer nivel al concurrir con el sistema tradicional de la justicia ordinaria. Reconociendo normativamente las costumbres, valores, tradiciones y respetando el derecho consuetudinario de las comunidades existentes.

Por otro lado, con relación a las decisiones que toma la justicia de paz al momento de resolver los conflictos ello guarda relación con los valores y principios culturales y sociales de la población en que se inserta el juez antes que, en la formalidad de la ley, lo que conduce a que la evaluación de estas decisiones deba responder a factores distintos a los que se utiliza en la justicia formal.

De ahí que una característica de la actuación de la justicia de paz es la no-formalización del conflicto, en tanto que el manejo de este suele efectuarse no estructurado - sin etapas fijas- y de manera oral. Sin embargo, debemos puntualizar que esta es una práctica común de estos jueces al expresar en actas escritas los acuerdos o decisiones que se toman en cada caso. De esta manera, el funcionamiento de esta justicia suele contar con un registro escrito que permite evaluar y analizar sus decisiones a posterior. Estos registros han permitido conocer el papel determinante que tienen las costumbres derecho consuetudinario-y prácticas locales en las decisiones de la justicia de paz, lo que conduce a que en la mayor parte de casos los jueces jueguen el rol de establecer el orden y la "armonía" en las relaciones sociales vigentes.

Otro aspecto importante es con relación a los cambios que se vienen dando respecto la justicia de paz es del tipo de persona que accede al cargo de juez de paz, a partir de mediados de los noventa en adelante, se nota un impacto importante respecto del tipo de prácticas y decisiones de la justicia en cada comunidad. Por un lado, cada vez es mayor el número de jóvenes con educación que se integran a la justicia de paz, especialmente en la costa; por otro lado, la presencia de las mujeres ha trascendido en los cargos accediendo al cargo de juez de paz el cual se ha incrementado, llegando a la actualidad a un porcentaje significativo (14\%) del total de jueces de paz.

Igualmente podemos destacar que, por la proximidad cultural del Juez de Paz con su comunidad, éste constituye factor de confianza en la tramitación y solución de 'los conflictos. La aceptación popular de los magistrados de paz y el éxito de la conciliación promovida por los jueces de paz se hallan en directa relación con la no formalización del conflicto como característica definitoria de la actividad que éstos despliegan en cumplimiento de sus funciones jurisdiccionales. Su reconocimiento y garantía deriva del acceso que tienen los pobladores en este tipo de justicia por la accesibilidad y la rapidez en la solución obtenida del conflicto y la restauración de la armonía social. 
El fortalecimiento debe ser permanente por parte del Poder Judicial conjuntamente con la Academia de la Magistratura deben diseñar un Programa Nacional de Formación y Capacitación continua con los Jueces de Paz electos a nivel nacional. La misma que deberá desarrollarse sobre tres ejes temáticos: Constitución y Derechos Humanos: Con el fin de que conozcan la norma fundamental, los derechos humanos a fin de que promuevan el respeto a los mismos y no se vulneren por los miembros de la comunidad. Capacitación con las autoridades locales, existente en cada comunidad a fin de informar de las funciones y competencias de cada institución evitando interferencias al momento de conocer los conflictos que se presenten. Capacitación en Derecho Consuetudinario y criterios de Equidad: que deberá aplicar el juez de paz para resolver los conflictos con las limitaciones de no violar los derechos fundamentales de la persona y especialmente de los principios y garantías del debido proceso.

\section{REFERENCIAS BIBLIOGRAFICAS}

ARDITO VEGA, W. (2002). Justicia de Paz: Nuevas tendencias y tareas pendientes. Lima: Instituto de Defensa Legal.

BRANDT, HANS J. (1990). En nombre de la Paz Comunal. Un análisis de la Justicia de Paz en el Perú. Centro de Investigaciones Judiciales de la Corte Suprema de la República. Fundación Friedrich Naumann, $1^{\circ}$ Edición. Lima.

CARDENAS TORRES, P. (2002). Jueces de Paz: nuevo paradigma de justicia democrática. Editorial Legis. Santa fe de Bogotá.

COMISION ANDINA DE JURISTAS. (2004) Los problemas de la Justicia en el Perú: hacia un enfoque sistémico. Diagnóstico Interinstitucional.

COMISION ANDINA DE JURISTAS (1999). Gente que hace justicia: La Justicia de Paz. $1^{\circ}$ Edición, Lima.

CHUNGA LAMONJA, F. (1998). La justicia de paz en el Perú: Manual para jueces de paz. Grijley. Cuarta edición.

DELGADO MENENVEZ, M.A.(1995). La opción por la justicia de paz. La Constitución de 1993: Análisis y comentarios Lima CAJ, pp 139-152. 
GARCIA SAYAN, Diego. (1987). Jueces no Letrados: El cuento de la vaca y la justicia. La justicia de paz y el pueblo. Lima. Centro de Investigaciones Judiciales De la Corte Suprema de JusticiaFundación Friedrich Naumann. 1987, pp39-43

LEDESMA, NARVAEZ M. La Justicia de Paz en el Perú. Fondo Editorial Universidad Inca Garcilaso de la Vega. $1^{\circ}$ Edición, Lima.

LOBATÓN, D. y ARDITO, W. (2002). Justicia de Paz. Nuevas tendencias y tareas pendientes. Instituto de defensa Legal.

LOLl, (1993) S. "Historia, Derecho y Sociedad en el Perú, republicano del siglo XIX. Politeia N 12 , Facultad de Derecho y Ciencias Políticas de la Universidad Nacional de Bogotá. pp 201-220.

LÓPEZ, J. LUIS. "Manual de los Juzgados de Paz”, disponible en:

http://www.intercodex.com/MANUAL-DE-LOS-JUZGADOS-DE-PAZCD-

ROM_L9788470524301.html [consultado el 08/11/20]

Poder Judicial (2015). Protocolos para una justicia intercultural. Lima: onajup

PONCE SILÉN, CARLOS. (1996) “Sistemas alternativos y la justicia de paz en el mundo”. Unión europea Asociación Civil Primero Justicia. Caracas.

PUENTES, L. (1997). Juzgados de Paz en la Costa Norte del Perú. Centro de Estudios y Publicaciones.

PROGRAMA DE LAS NACIONES UNIDAS PARA EL DESARROLLO (PNUD). (2015). Fortaleciendo la Justicia de Paz en el Perú. Recuperado de https://www.pe.undp.org/content/peru/es/home/library/democratic_governance/fortaleciendola-justicia-de-paz-en-el-peru.html [Consulta: 4 de febrero 2020].

REVILLA, Ana Teresa (1995). "La Justicia de Paz en el Perú., en Justicia y DEAROOL: Agenda Para el siglo XXI. Bogotá. Ministerio de Justicia y del Derecho de Colombia. pp 135-166

REVILLA, Ana Teresa y PRICE, Jorge (1992). La administración de justicia informal: Posibilidades de integración. Lima Fundación M. J. Bastamente de la Fuente. 
SILES, Abraham (1990) La Justicia de Paz y su labor esencialmente conciliadora. Un análisis de Actas de Conciliación. Lima: Instituto de Defensa Legal.

UPRIMNY, RODRIGO. “Jueces de Paz y Justicia Informal: Una aproximación a sus potencialidades y limitaciones", disponible en:

http://redesalternativas.com.ar/noticias_ver.php?id=283, [consultado el 31/01/21].

Ugarte, J. (1987). Reseña histórica del origen y evolución de la justicia de paz en el Perú. En La justicia de paz y el pueblo (pp. 19-21). Lima: Fundación Friedrich Naumann y Centro de Investigaciones Judiciales de la Corte Suprema de Justicia de la República 